\title{
Meta
}

Journal des traducteurs

Translators' Journal

\section{Le langage, la poésie et la traduction poétique ou une approche scientifique de la traduction poétique}

\section{Erol Kayra}

Volume 43, numéro 2, juin 1998

URI : https://id.erudit.org/iderudit/003295ar

DOI : https://doi.org/10.7202/003295ar

Aller au sommaire du numéro

Éditeur(s)

Les Presses de l'Université de Montréal

ISSN

0026-0452 (imprimé)

1492-1421 (numérique)

Découvrir la revue

Citer cet article

Kayra, E. (1998). Le langage, la poésie et la traduction poétique ou une approche scientifique de la traduction poétique. Meta, 43(2), 254-261. https://doi.org/10.7202/003295ar
Résumé de l'article

L'auteur discute de divers aspects de la traduction poétique. Plus qu'un simple recodage, la traduction poétique est un art en soi, une création originale, une activité pratique et de savoir lié à la perception et à la création. 


\title{
LE LANGAGE, LA POÉSIE ET LA TRADUCTION POÉTIQUE OU UNE APPROCHE SCIENTIFIQUE DE LA TRADUCTION POÉTIQUE ${ }^{1}$
}

\author{
EROL KAYRA \\ Université Çukurova, Adana, Turquie
}

\begin{abstract}
Résumé
L'auteur discute de divers aspects de la traduction poétique. Plus qu'un simple recodage, la traduction poétique est un art en soi, une création originale, une activité pratique et de savoir lié à la perception et à la création.
\end{abstract}

Abstract

This article discusses various aspects of poetic translation. An art in itself and a form of creativity, poetic translation constitutes more than language to language transfer; it is an activity involving both perception and creation.

\section{INTRODUCTION}

La traduction poétique est d'abord, à mon sens, un art de recoder, c'est-à-dire une activité linguistique destinée à déchiffrer les codes d'un message source et à produire par voie de transfert de sens et de style sa double cible, dont les constituants sont reliés à ces mêmes codes par une relation à la fois interne et externe. En effet chaque mot, ou chaque groupe de mots, prend son sens véritable selon sa position contextuelle ou sa fonction dans l'énoncé dont il est un constituant. C'est dire que l'activité de traduction poétique ne consiste pas seulement à transférer d'une langue à l'autre une pensée ou un sentiment, mais aussi à mettre en œuvre une valeur d'ordre esthétique mais de caractère sonore. En d'autre termes, le langage poétique, en tant qu'il implique une double structure, ou plus exactement une double dimension d'interdépendance - dimension référentielle et dimension stylistique ou esthétique (Hymes 1984: 26) quant à sa structure interne et à sa valeur sonore - peut se définir comme une expression ou une transposition au moyen de «signes particuliers» (Bréal 1904 : 329) d'une psychologie ou d'un comportement, d'un sentiment ou d'une pensée, d'une attitude ou d'une esthétique. D'où la différence entre le mode poétique fondé sur la fonction symbolique et le pouvoir harmonique du langage, et le mode technique ou prosaïque fondé sur des codes dont la fonction ne consiste qu'à signifier et à communiquer une expérience objectivante et rationnelle (Guiraud 1971), expliquée tout simplement par cette connaissance du monde et du réel découpé différemment dans chaque langue, et cela non seulement parce qu'il s'agit pour chaque langue de rapports différents à «exprimer» (Martinet, cité par Mounin 1963 : 261), mais aussi parce que «chaque langue est constamment en formation et en développement» (Gadamer $1975: 415)$. Or le code poétique ne consiste pas seulement à nous informer, mais aussi et surtout à nous communiquer une certaine impression, à provoquer en nous des «émotions analogues» (Earnap, Philosophy and Logical Syntax), et cela non seulement par le pouvoir symboli-

Meta, XLIII, 2, 1998 
que des mots qui impliquent des sens au-delà de leur aire naturelle (Lyons 1970), mais aussi par leur valeur harmonique.

\section{ACTIVITÉ DE TRADUCTION}

\subsection{La traduction poétique est une activité aux multiples dimensions}

C'est de là que vient ce caractère symbolique, expressif, descriptif et stylistique de l'œuvre poétique dû, d'une part, à la vertu des codes choisis parmi les plus abtraits et dont on utilise toutes les nuances et, de l'autre, au pouvoir imaginatif et harmonique des figures, qui ont une fonction très variée - descriptive, combinatoire, expressive, codificatrice, antithétique, etc. - ce qui fait de la traduction poétique une activité aux multiples dimensions.

De là on peut déduire que, dans la traduction poétique, les codes, étant de nature non seulement à actualiser les valeurs connotatives mais aussi à les développer (Guiraud 1971), se trouvent canalisés nécessairement autour d'une image-concept (Claret 1979) et prennent leur véritable signification eu égard à cette image-concept qui tient au contenu sémantique du contexte en même temps qu'à la valeur phonique des éléments utilisés en vue d'augmenter l'efficacité du pouvoir poétique et d'exercer ainsi une influence relative sur la substance sémantique. C'est dire que les éléments intrinsèques qui caractérisent l'œuvre poétique tels que couleur, harmonie, rythme, ont un caractère plastique et sont de nature à désigner un rapport interne et constitutif entre le sens et le son considéré par certains linguistes (Hope, par exemple) comme un «un écho du sens». Mallarmé, quand il disait qu'on fait des vers avec des mots, ne voyait pas les mots, certes, comme de simples substituts sémantiques; il entendait plutôt par-là l'harmonie des mots, c'est-à-dire cette parfaite convenance entre le son et le sens, et voulait nous amener ainsi à avoir une logique et une esthétique de la poésie où l'idée et le symbole se trouvent constamment associés. Je veux dire par là que la traduction poétique n'est pas une simple opération d'ordre lexical; elle est une activité linguistique prise sous toutes ses formes en même temps qu'une esthétique de caractère phonique impliquant le sens le plus mélodique du mot. Ce rapport strict entre la poésie et le langage fait de la poésie un art du langage, et du traducteur un bon technicien du langage mais conscient de l'effet poétique qui est au fond l'œuvre commune du sens et du son.

\subsection{La traduction poétique est une création originale}

Il s'ensuit que l'esprit véritable de la traduction poétique est impliqué dans la définition même de la poésie, qui peut être considérée comme un art de la parole destiné à créer une émotion et un style propres à lui et qui n'existent pas dans d'autres combinaisons verbales ou cas du langage, en ce sens que la parole est prise ici en tant qu'unité de langage à double valeur : la valeur représentative ou symbolique, celle qui constitue une image et un tableau, et la valeur communicative, celle qui consiste à transmettre un message fait plus souvent de l'extérieur vers l'intérieur et impliquant l'idée poétique, c'est-à-dire cette idée «qui mise en prose, réclame encore le vers» (Valéry 1941 : 44). Cette caractéristique de la poésie, et par conséquent du langage poétique, nous conduit à être soucieux de ce qui est rythmique et concentrique linguistiquement et esthétiquement parlant, à traiter le texte source à deux niveaux (niveau d'ordre référentiel et niveau d'ordre stylistique ou esthétique) et sur deux plans (plan sensible et plan intelligible), parce qu'il s'agit de concilier dans une même expérience Idée et Harmonie, qui ont leur source «dans les variations stylistiques et connotatives» (Guiraud 1971:10) issues des propriétés formelles et substantielles des figures, tropes, métaphores, etc. (Kayra 1993 : 164). 
Compte tenu de cette caractéristique du langage poétique où les significations sont même saisies, comme le dit Jean Rousselot, «à travers des formes» (1962), la tâche première du traducteur poétique doit consister à poser le problème, d'une part, au niveau de la fonction poétique elle-même et, de l'autre, au niveau où le message poétique (le vers) est pris en tant que partie de la linguistique, car il s'agit avant tout d'une série bien combinée d'énoncés qui demandent à être analysés sur le plan sémiotico-sémantique.

Il faut donc traiter d'abord le message poétique au regard des éléments propres à la poésie qui consistent en deux aspects - l'aspect acoustique et phonétique, révélé par l'expression et qui constitue la substance phonique, et l'aspect sémantique lié aux significations et qui constitue la substance du contenu - et l'analyser ensuite en tant que «forme conventionelle et strictement codifiée du langage» (Cohen $1966: 8$ ). Cela revient à dire qu'il ne suffit pas de traduire le sens, qu'il faut traduire aussi le style ou l'esthétique du poète qui procède de son sens de la beauté formelle et de la profondeur psychologique. Jakobson voit même dans le style un élément servant de sous-code par rapport au code total. On lui attribue même une fonction supra-segmentale faisant partie du message communiqué (Hill 1958). Ce n'est pas par hasard qu'on emploie le terme «poétiser» pour exprimer l'idée d'esthétique et de musical. Dépoétiser quelque chose n'est-ce pas lui faire perdre l'harmonie et le rythme intérieur, lui enlever la musique et l'esthétique ? Par musique poétique, il ne faut sans doute pas entendre une simple sonorité verbale; elle est sonorité suggestive, car il s'agit de produire un effet d'orchestration et d'ajouter à un mot une signification que ce mot ne porte pas en lui-même substantiellement. C'est même de cette fusion Idée-Musique que provient ce caractère hermétique du message poétique.

\subsection{La traduction poétique est une activité pratique et de savoir}

Exprimer la même chose dans une autre langue sans rien perdre de sa musique et de son harmonie, de sa couleur et de son rythme intérieur exige nécessairement une pratique linguistique et péri-linguistique en même temps qu'une série de compétences de caractère spécifique, entre autres une spécialisation du domaine, un sens de l'harmonie et du rythme, et surtout ce goût du beau poétique, qui réside tant en la forme qu'en le fond, en le visible qu'en le caché. Le traducteur est un «co-auteur» animé d'une certaine théorie esthétique mais doté en même temps d'une certaine pédagogie de la pratique en son genre. Le plus pratique n'est-ce pas «le plus rationnel»? (Barthes $1970: 47)$. Le traducteur doit savoir aussi actualiser l'atmosphère du poème, sinon son sujet, sans porter atteinte sans doute à son contenu sémantique, à sa valeur phonique et à sa dimension universelle. Le traducteur, à mon sens, peut ajouter une certaine couleur au poème qu'il traduit mais sans faire abstraction du caractère propre à la création poétique.

Tout cela nous montre qu'il faut développer pour chaque poète, du moins pour chaque genre poétique, une théorie de la traduction. Prenons la poésie surréaliste, où l'on utilise un langage «décomposé» fondé sur «le jeu désintéressé de la pensée», sur «l'automatisme verbo-visuel» (Breton 1934), et la poésie de Valéry, où nous sommes en présence d'une structure abstraite de la réalité vécue, saisie et interprétée à travers et par l'intuition : il faut traduire la poésie surréaliste de façon à conserver son caractère philosophique et son aspect pathétique, alors que dans la poésie valéryenne, l'attention doit porter sur les symboles et les images riches d'impressions et de suggestions, sans négliger «sonorités, timbres, accents» (Varéry 1941: 99), car il s'agit d'une poésie vue comme «un système intelligible et imaginable de l'expression» (Varéry 1941: 178) dont l'essentiel résiderait en «le beau son» (Varéry 1941 : 182). En effet, chez Valéry, où l'on utilise plusieurs dénominations pour un même concept (le «corps», par exemple, y est dénommé «ma triste beauté», «ma chair de lune et de beauté», «mortelle sœur», «chair maîtresse»), nous assistons à une création poétique impliquant l'idée d'harmonie et de réciprocité entre 
idées, impressions, impulsions et moyens d'expression. Le moi y est laissé se découvrir à travers son double présenté au même titre que l'universel pur et dépouillé ainsi de toute contingence. Il s'agit, pour ainsi dire, d'un travail sur soi destiné à retrouver ce qu'il y a de plus profond et de plus absolu dans le Moi, d'une interprétation purement symbolique du monde saisi par une conscience conquise à la mesure d'une s'agesse dépouillée de son sens ordinaire. Le poète cherche, à cet effet, à donner aux images, figures, métaphores, etc., outre leur propre signification, une signification susceptible de donner une interprétation originale de l'univers. La fontaine, par exemple, quoiqu'elle symbolise le mortel, n'est qu'un miroir où se reflète la figure «éternellement pure» de Narcisse, symbole de la caresse, c'est-à-dire de la tendresse que le poète n'avait pas pu trouver chez sa mère. En l'arbre, nous avons la représentation symbolique de la destinée humaine :

Ils vivent séparés, ils pleurent confondus Dans une seule absence

Et leurs membres d'argent sont vainement fendus À leur douce naissance.

(Charmes, «Au platane», pp. 42-43)

D'où, dans la traduction poétique, la bonne utilisation des figures, entre autres des métaphores et des métonymies, qui peuvent être confondues, car dans le langage poétique, pour reprendre les termes de Jakobson, «toute métonymie est légèrement métaphorique et toute métaphore a une teinte métonymique» $(1983: 238)$. D'où le bon choix des éléments susceptibles d'ajouter une valeur poétique tant au niveau des sonorités et des rythmes que des images, tels que homophonie, assonance, redondance. D'où aussi cette conscience à l'égard du principe de similarité et de contraste sémantiques, susceptibles d'être apportés par le parallélisme métrique et l'équivalence phonique (Jakobson 1963 : 66-67), sans passer sous silence le rôle joué par des figures utilisées en vue de servir de support aux significations, telles que archaïsmes, étymologismes, néologismes. À titre d'exemple, pour «images», Valéry utilisait «idoles».

Le traducteur d'autre part, doit savoir, comme le poète, tirer des effets sonores en recourant à la force de leur suggestion acoustique. Prenons le cas des onomatopées ou des autres inventions ajoutées à ces onomatopées et qui consistent en de simples jeux de sonorités pures. Dans cet effort, il est préférable que le traducteur recourre à la vertu des éléments formels pouvant donner la sensation d'une idée ou d'un sentiment plutôt que de chercher à donner aux sons le sens du mot dans lequel ils sont introduits. C'est dire qu'il faut considérer le sens, l'harmonie, le rythme comme les aspects différents mais complémentaires d'une réalité poétique. L'important, c'est de pouvoir bien saisir le sens profond et symbolique du message poétique et d'être conscient de la valeur contextuelle des éléments utilisés en vue de créer l'effet poétique. Prenons Valéry et Éluard qui ont des traits communs. Le premier aime bien recourir à l'opposition des périphrases vocatives, tandis que le second se contente de la fonction apostrophique des périphrases métaphoriques (Suhamy 1992). Dans la traduction des images (métaphores ou comparaisons), dont le nombre montre bien la dimension psycho-imaginative de la création poétique, il faut donc recourir à un procédé de codage qui convient à l'emploi de ces images, c'est-à-dire qu'il faut les traiter selon qu'elles jouent un rôle expressif, hypothétique, ou bien chimérique.

Il y a donc dans la poésie des constantes et des variables. Le traducteur doit partir des constantes pour retrouver les variables. Dans l'étude des significations, par exemple, il faut mettre en œuvre deux processus de caractère opposé mais complémentaire (Claret 1979) :

- le processus qui se rapporte au signe, c'est-à-dire celui qui consiste à déterminer le concept en partant du signe et qu'on peut appeler l'étude sémasiologique; 
- le processus qui tient à l'idée, c'est-à-dire celui qui consiste à étudier les dénominations en partant du concept et qu'on peut appeler l'étude onomasiologique.

À cause des différences stylistiques et métaphoriques et de la fréquence des images allusives, il n'est pas facile d'aller à une équivalence idéale. Pour ne pas tomber dans l'illusion, il vaut mieux reformuler un mot du texte source à la lumière de son interprétation contextuelle que de chercher dans la langue cible son équivalence sémantique. Et, d'autre part, le poète peut recourir aux jeux du langage destinés à nous éveiller à la conscience d'une réalité à peine perceptible. Dans ce cas, le traducteur peut avoir du mal à distinguer le rapport susceptible d'exister entre figures et idées, c'est-à-dire entre l'objet réduit à ses propriétés essentielles et l'idée qui en fait une réalité symbolique. L'important, pour le traducteur, c'est de saisir l'idée centrale et de la reformuler par les mots correspondants de la langue cible mais sans perdre l'humour et le style du texte source. Prenons ces vers de Valéry :

Le vent se lève !... Il faut tenter de vivre !

L'aire immense ouvre et referme mon livre,

La vague en poudre ose jaillir des rocs!

Envolez-vous, pages tout éblouies !

Rompez, vagues ! Rompez d'eaux réjouies

Ce toit tranquille où picoraient des focs !

(Le cimetière marin, Poésies, $1942: 151$ )

Le traducteur doit décomposer les vers précités non pas selon leurs unités verbales mais selon les concepts qui y sont inclus, pour éviter la confusion susceptible de procéder de la complexité et de l'ambiguïté des idées et des images évoquées dans le poème, faute de quoi il risque d'entendre à sa manière le poème et de reconstituer ainsi l'univers poétique à son gré. C'est dire qu'il faut saisir l'idée poétique dans sa pureté originelle. Le mot «toit», qui a normalement double sens : couverture d'une maison (sens dénotatif) et abri, foyer (sens connotatif), désigne dans le texte «mer» où «picorent» les focs comparés par analogie aux oiseaux. Autrement dit, entre le mot «toit» et le mot «picorer», sémantiquement parlant, il y a un rapport dénotatif, parce que «picorer» est un terme utilisé pour les oiseaux et les oiseaux picorent sur le toit. Mais sur le plan symbolique, entre ces deux termes, il y a un rapport de caractère allusif destiné à mettre en valeur un développement psychologique d'ordre intuitif, qui va d'une réalité concrète à une réalité jugée sur le plan de l'idée. Cela revient à dire qu'il faut partir non pas des mots mais des images qui s'en dégagent. Prenons le vers «Ce toit tranquille où picoraient des focs !». Si l'on reconstruit le même vers avec des mots considérés dans leur valeur descriptive normale, il devrait, si j'ose dire, être recomposé comme suit : «La mer tranquille où nageaient des focs !».

\subsection{La création poétique est un acte de voir d'abord et de créer ensuite}

Jugée au même titre que la poésie, c'est-à-dire vue en tant qu'expérience destinée à atteindre au beau esthétique par les effets du langage, qui est à la fois idée (sens) et musique (son), «la traduction poétique ne peut donc être qu'un acte de voir d'abord et de créer ensuite mais à partir d'un contenu qui va du clair au sombre, du concret à l'abstrait, et conçu d'après une esthétique au sens étymologique du mot, c'est-à-dire prise dans le sens d'aesthetica, qui signifie sentir et par analogie "avoir le sentiment du beau"» (Kayra 1993). En d'autres termes, il faut pouvoir apporter une juste explication aux symboles, images et autres formes du langage poétique, qui «se lie[nt] au moins comme double» (Kristeva 1969 : 146) et qui a une émotion à laquelle participent le rythme, l'harmonie et «la musique avant toute chose» (Verlaine 1975: 25). Il faut pour cela remonter aux sources du poème pour y chercher le véritable charme poétique, atteindre «le suggestif symbole» 
(Ghil 1887). Cela suppose, pour ainsi dire, la recherche d'une syntaxe picturale liée aux lois de l'esthétique musicale (Saoula : 1969) et l'utilisation d'un langage fondé sur l'esprit d'analyse et de synthèse. Le passage d'une langue à l'autre n'est pas seulement une opération d'analyse mais aussi de synthèse, car, particulièrement dans la traduction poétique, il s'agit de fixer non seulement l'origine des obstacles rencontrés dans les deux langues mais aussi l'élément commun qui provient de ces obstacles.

Il faut donc voir dans le concept non pas simplement la signification d'une réalité perçue par la conscience réfléchie, mais aussi une dynamique, ou plutôt une explication symbolique et indirecte du moi plié à tout développement psychologique, un reflet verbal d'un moi saisi jusqu'à ses dimensions cosmiques. Car le concept en poésie est plus qu'une chose; il est «quelqu'un pensant à cette chose» (Valéry, cité par Claret 1979: 10). Il n'intervient pas seulement comme un abstrait de la vérité, il la décompose aussi (Gusdorf 1953). Cela vient du fait que le code poétique a affaire à l'expérience affective fondée sur le redoublement du sens, et met en jeu un procédé qui va jusqu'à déstructurer le message à cause d'un type de relations qui est différent de celui que présentent les processus formels et sémantiques habituels. Il s'agit, certes, «de la même quantité de réalité» (Mounin 1963 : 42), mais d'une combinaison verbale différente de cette réalité, car la même idée peut être exprimée sous plusieurs formes, ce qui permet d'ajouter ou d'ôter du moins une certaine nuance à la signification du code. D'où dans la traduction poétique cet effort relatif au caractère dichotomique des codes poétiques considérés sous tous leurs aspects et à tous leurs niveaux : signifiant et signifié, forme et substance, sens dénotatif et sens connotatif, sens et références, fonction d'allusion et fonction de prolongements du symbole, signification générale et signification contextuelle, bref cette étude approfondie de la fonction poétique analysée tant au niveau de la linguistique qu'au niveau de la poétique prise dans son sens étymologique, poiêtikê, qui signifie teknê, c'est-à-dire technique de la poésie. C'est alors qu'il est possible d'arriver à une analyse juste et claire des concepts et par là à un équivalent plus proche du texte source. Car l'activité de traduction poétique, considérée à juste titre comme une sorte de «sémiologie pratique», repose au fond, pour reprendre les termes de Valéry, «sur un travail de purification des mots et des idées» (Cahiers, II, 788), parce qu'il est question d'identifier, du moins de concilier soit au niveau du concept, soit au niveau de l'énoncé (le vers) le texte source et le texte cible formés d'éléments d'une nature à susciter des modes différents de saisir une réalité souvent subjective. Le traducteur doit donc avant toute chose être concient du fait que la structure sémiotico-sémantique du langage poétique n'est pas seulement de caractère expressif, séquentiel, relationnel ou fonctionnel, mais qu'elle est aussi de caractère relatif, essentiellement lorsqu'il s'agit d'une poésie impliquant une infinité de messages, de caractère souvent hermétique.

Ce caractère polymorphe et polyvalent du langage poétique nous conduit à étudier le problème de traduction poétique sur trois plans essentiels, à savoir :

1) le plan psychologique ou sensible,

2) le plan logique ou intelligible,

3) le plan relationnel ou fonctionnel,

qui correspondent chacun à une conscience différente :

a) Conscience intellectuelle créé par l'attention centrée sur le contenu sémantique des concepts (stade de compréhension linguistique au niveau des concepts). D'où le rôle du savoir dans la traduction poétique.

b) Conscience affective ou intuitive créée par la communion directe entre le poète et le traducteur (stade d'assimilation qui consiste à voir comme le poète). D'où le rôle du sensible et du comportement dans la traduction poétique. 
c) Conscience pratique destinée à situer le traducteur sur le même plan que celui du poète (stade de fabrication, ou de production, qui consiste à créer comme le poète). D'où le rôle de la création potentielle dans la traduction poétique.

Chacune de ces consciences requiert nécessairement une certaine compétence, qui peut être énumérée comme suit :

1) compétence d'ordre linguistique au sens le plus large du terme,

2) compétence d'ordre comportemental,

3) compétence d'ordre potentiel.

Il en résulte que la traduction poétique est :

a) une activité saisie au niveau du concept,

b) un comportement individuel, qui relève, d'une part, de l'étoffe psychique ayant sa source dans la psychologie individuelle et dans l'intuition créatrice et, de l'autre, de la compétence esthétique rattachée à ce qu'on peut appeler goût (auditif, poétique, etc.),

c) une expérience qui vise à produire une émotion dite poétique, qui est différente des émotions de la vie réelle et qui est de caractère immanent, c'est-à-dire potentiel.

\section{CONCLUSION}

En conclusion, dans la traduction poétique, il faut commencer par voir la poésie comme la pensée la mieux organisée tant du point de vue linguistique que du point de vue esthétique, ou comme l'expérience sensible la plus hermétique. Cela est nécessaire pour être dès le début conscient du fait que la traduction poétique n'est pas exclusivement une activité faite sur le seul plan linguistique. En tant qu'elle est un acte de voir d'abord et de créer ensuite d'après un modèle qui nous montre à peine sa figure propre, elle devient nécessairement à la fois un art et une technique où interviennent nombre de compétences, qui vont du symbolisme linguistique au symbolisme phonologique. Elle est un art, parce qu'elle implique une originalité marquée par une certaine esthétique. Elle est une technique, parce qu'elle a ses règles et son esprit, conçus à la lumière des connaissances et des techniques acquises empiriquement. Cette caractéristique de la traduction poétique nous conduit forcément à procéder à une analyse juste et claire des problèmes posés par les contraintes susceptibles de provenir soit de l'art poétique proprement dit, soit du caractère descriptif et analytique du langage. Une telle analyse ne nous permet pas seulement de mieux apprécier la juste valeur du contenu connoté dans le texte source, d'aborder le problème dans sa perspective «sémiotico-sémanticiste» (Ladmiral 1979) et sa valeur stylistique, mais aussi de l'étudier plus consciemment et plus nettement sur son plan herméneutique qui se trouve à l'origine de l'analyse poétique. Elle permet, d'autre part, de saisir la nature et la dimension exactes de la création poétique, de mettre en œuvre une pratique traduisante jugée à tous les niveaux et de voir les choses de l'œil à la fois d'un théoricien et d'un praticien, chose nécessaire pour une activité de cette ampleur.

\section{Note}

1. Communication présentée au $\mathrm{XV}^{\mathrm{e}}$ Congrès International des Linguistes, Université Laval, Québec (Canada), les 9-14 août 1992, Secton 12 : Théories du langage. Il s'agit ici d'une version partiellement modifiée de ladite communication publiée dans : Les Actes du XV Congrès International des Linguistes, Presses de l'Université Laval, Québec (Canada), 1993. 


\section{RÉFÉRENCES}

ABASTADO, C. (1980) : Messages des médias, Paris, CEDIC.

BARTHES, R. (1957) : Mythologies, Paris, Éditions du Seuil.

BARTHES, R. (1970) : L'empire des signes, Genève, Éditions Skira.

BEDOUIN, J.-L. (1964) : La poésie surréaliste, Paris, Seghers.

BRÉAL, M. (1904) : Essai de sémantique, Paris, Hachette.

BRETON, A. (1934) : «Le message automatique», Point du jour.

CARY, E. (1985) : Comment faut-il traduire ?, Lille, Presses Universitaires de Lille, 94 p.

CLARET, J. (1979) : L'idée et la forme, Paris, PUF, 127 p.

COHEN, J. (1966) : Structure du langage poétique, Paris, Flammarion.

Earnap, Philosophy and Logical Syntax, cité dans J. Cohen (1966), Structure du langage poétique, 194 p.

GADAMER, H.-G. (1975) : Truth and Method, New York, Sheed and Ward.

GHIL, R. (1887) : Traité du verbe, cité dans Henri Lemaître, La poésie depuis Baudelaire, Paris, Armand Colin, coll. «U», 1965.

GUIRAUD, P. (1956) : «Les champs morpho-sémantiques», Paris, BSL, fas. 1, pp. 265-288.

GUIRAUD, P. (1971) : La sémiologie, Paris, PUF.

GUSDORF, G. (1953) : La parole, Paris, PUF.

HILL, A. (1958) : Introduction to Language Structures, New York, Harcourt, Brace and World.

HYMES, D.H. (1984) : Vers la compétence de communication, Paris, Crédit-Hatier, coll. «LAL».

JAKOBSON, R. (1963) : Essais de linguistique générale, t. 1, Paris, Les éditions de Minuit, 260 p.

KAYRA, E. (1993) : «La traduction poétique : Exemples empruntés à Paul Valéry et Yunus Emre», Diogène, no 164, oct.-déc., pp. 80-90.

KRISTEVA, J. (1969) : Recherches pour une sémanalyse (Extraits), Paris, Seuil, coll. «Points», 320 p.

KRISTEVA, J. (1981) : Le langage, cet inconnu, Paris, Seuil.

LADMIRAL, J.-R. (1979) : Traduire : théorèmes pour la traduction, Paris, Payot, 366 p.

LYONS, J. (1970) : Linguistique générale, trad. franç., Paris, Larousse.

MARTINET, A. (1960) : Éléments de linguistique générale, Paris, A. Colin.

MOUNIN, G. (1963) : Problèmes théoriques de la traduction, Paris, Gallimard, coll. «TEL».

MOUNIN, G. (1964) : La Machine à traduire, Paris, La Haye, Mouton, 212 p.

ROUSSELOT, J. (1962) : Forme et Signification: essai sur les structures littéraires de Corneille à Claudel, Paris, J. Corti.

ROUSSELOT, J. (1969) : Mort et survie du langage, Paris, Bruxelles, Sodi.

SAOULA, C. (1969) : Gloses sur Mallarmé, Paris, Diderot, cité dans J. Rousselot, Mort et Survie du langage.

SUHAMY, H. (1992): Les figures du style, Paris, PUF, 128 p.

VALÉRY, P. (1941) : Tel Quel, I, Paris, Gallimard.

VALÉRY, P. (1942) : Poésies, Paris, Gallimard.

VALÉRY, P. (1952-1963) : Euvres, Paris, Gallimard, coll. «Bibliothèque de la Pléiade», édition établie par Jean Hytier.

VERLAINE, P. (1975) : Jadis et Naguère, «Art poétique», Paris, Librairie Générale Française.

Colloque sur la traduction poétique (1978) : Paris, Gallimard, 314 p. 